\title{
FOURIER TRANSFORMS AND THE HERMITE-BIEHLER THEOREM
}

\author{
G. CSORDAS AND R. S. VARGA
}

(Communicated by Irwin Kra)

\begin{abstract}
A new necessary and sufficient condition for real entire functions, represented by Fourier transforms, to have only real zeros is proved. An application of this result to the Riemann $\xi$-function is also given.
\end{abstract}

\section{INTRODUCTION}

The purpose of this paper is (1) to establish necessary and sufficient conditions for real entire functions, represented by Fourier transforms, to have only real zeros and (2) to apply this result to the Riemann $\xi$-function. The present investigation is a continuation of the work that began with the researches of Pólya ([P1], [P2]) and de Bruijn [B] (cf. also [CNV], [CV1], [CV2], and [CV3]).

In Section 2, we review some pertinent definitions and provide the statements of the results required in Section 3. An important assumption in the main theorem (cf. Theorem 3.4) is that all the zeros of the entire function

$$
F(x ; K):=\int_{-\infty}^{\infty} K(t) e^{i x t} d t \quad(x \in \mathbf{C}),
$$

lie in a horizontal strip, where $K(t)$ in (1.1) is an admissible kernel (cf. Definition 2.2). The interesting and useful aspect of this result (Theorem 3.4) is that it combines this geometric condition on the location of the zeros of $F(x ; K)$ with the classical conditions of Jensen (Theorem 2.2) and Pólya (Theorem 2.4). Our method of proof is based on the Laguerre inequalities (Theorem 2.1), which are satisfied by functions in the Laguerre-Pólya class (cf. Definition 2.1), and the Hermite-Biehler theorem for entire functions (Theorem 2.5). With the aid of the Hermite-Biehler theorem, we generate two families of functions in the Laguerre-Pólya class and we express a classical necessary and sufficient condition for an entire function to have only real zeros (Theorem 2.4) in terms of these functions, using a crucial technical result (Lemma 3.3). Finally, we use

Received by the editors October 3, 1988.

1980 Mathematics Subject Classification (1985 Revision). Primary 30D10, 30D15.

Key words and phrases. Fourier transforms, Laguerre-Pólya class, Laguerre inequalities, Hermite-Biehler theorem, Riemann Hypothesis, Riemann $\xi$-function.

This research was supported, in part, by the National Science Foundation under Grant DMS8713737 
the foregoing results and the known properties of the Riemann $\xi$-function (cf. (3.17)), to obtain (Corollary 3.5) a new condition for the Riemann Hypothesis to hold.

\section{DEFINITIONS AND PRELIMINARY THEOREMS}

For the reader's convenience, we review here the definitions and theorems which will be needed in the statements and in the proofs of the new results of Section 3.

Definition 2.1. A real entire function $f(x)$ is said to be in the Laguerre-Pólya class, written $f(x) \in \mathscr{L}-\mathscr{P}$, if $f(x)$ can be expressed in the form

$$
f(x)=C e^{-\alpha x^{2}+\beta x} x^{n} \prod_{j=1}^{\omega}\left(1-x / x_{j}\right) e^{x / x_{j}} \quad(\omega \leq \infty),
$$

where $\alpha \geq 0, \beta$ and $C$ are real numbers, $n$ is a nonnegative integer and the $x_{j}$ 's are real and nonzero with $\sum_{j=1}^{\omega} x_{j}^{-2}<\infty$.

A necessary condition for a real entire function $f(x)$ to be in the LaguerrePólya class is that it satisfy the Laguerre inequalities (2.2).

Theorem 2.1 (The Laguerre Inequalities (cf. [L] or [S])). If $f(x) \in \mathscr{L}-\mathscr{P}$, then

$$
\left[f^{(m)}(x)\right]^{2}-f^{(m-1)}(x) f^{(m+1)}(x) \geq 0 \quad(x \in \mathbf{R} ; m=1,2,3, \cdots) .
$$

For a detailed proof of the following theorem of Jensen $[\mathrm{J}]$, see [CV3, Theorem 2.10].

Theorem $2.2([\mathrm{~J}])$. Set

$$
f(z):=e^{-\alpha z^{2}} f_{1}(z) \quad(\alpha \geq 0, f(z) \not \equiv 0),
$$

where $f_{1}(z)$ is a real entire function of genus 0 or 1 . Then $f(z) \in \mathscr{L}-\mathscr{P}$ if and only if

$$
\left|f^{\prime}(z)\right|^{2} \geq \operatorname{Re}\left\{f(z) \overline{f^{\prime \prime}(z)}\right\} \quad \text { for all } z \in \mathbf{C} .
$$

Before we apply Theorem 2.2 to real entire functions which are represented by Fourier transforms, we introduce the following definition.

Definition 2.2. A function $K: \mathbf{R} \rightarrow \mathbf{R}$ is called an admissible kernel, if it satisfies the following properties:

(i) $K$ is integrable over $\mathbf{R}$,

(ii) $K(t)>0 \quad(t \in \mathbf{R})$,

(iii) $K(t)=K(-t) \quad(t \in \mathbf{R})$, and

(iv) for some $\varepsilon>0, K(t)=O\left(\exp \left(-|t|^{2+\varepsilon}\right)\right)$, as $t \rightarrow \infty$.

The Fourier transform of an admissible kernel is a real entire function. More precisely, Pólya [P1] proved the following theorem. 
Theorem 2.3 ([P1], [P3]). Set

$$
F(x ; K):=\int_{-\infty}^{\infty} K(t) e^{i x t} d t,
$$

where $K(t)$ is an admissible kernel. Then $F(x ; K)$ is a real entire function of order $\rho:=\rho(F(x ; K))$, where, for some $\varepsilon>0(c f .(2.4)(\mathrm{iv})), \rho$ satisfies

$$
\rho \leq \frac{\varepsilon+2}{\varepsilon+1}<2
$$

While the problem of characterization of the admissible kernels, $K(t)$, whose Fourier transform $F(x ; K)$ (cf. (2.5)) belongs to the Laguerre-Pólya class, is a long-standing open problem (cf. [CV2]), one can apply Theorem 2.2 to $F(x ; K)$ and obtain the following necessary and sufficient conditions.

Theorem 2.4 ([P2, p. 17, formula (18)]). The function $F(x ; K)$ defined by $(2.5)$ is in the Laguerre-Pólya class if and only if

$$
\Delta(x, y ; K) \geq 0 \quad \text { for all } x, y \in \mathbf{R},
$$

where

$$
\Delta(x, y ; K):=\int_{-\infty}^{\infty} \int_{-\infty}^{\infty} K(t) K(s) e^{i(t+s) x} e^{(t-s) y}(t-s)^{2} d t d s
$$

In formulating an extension of the classical Hermite-Biehler theorem to (transcendental) entire functions (cf. [Le, Chapter VII]), it will be convenient to adopt the following definition (cf. $[\mathrm{AK}]$, [Le] or $[\mathrm{M}]$ ).

Definition 2.3. An entire function $\omega(z)$ is said to be a function in class $\overline{H B}$ if

(i) $\omega(z)$ has no zeros in the open lower half-plane, i.e.,

$$
\omega(z) \neq 0 \quad \text { if } z \in H^{-}:=\{z \in \mathbf{C}: \operatorname{Im} z<0\},
$$

and

$$
\left|\frac{\omega(z)}{\bar{\omega}(z)}\right| \leq 1 \quad \text { for } \operatorname{Im} z>0,
$$

where $\bar{\omega}(z)$ denotes the entire function obtained from $\omega(z)$ by replacing the Maclaurin series coefficients of $\omega(z)$ by their conjugates.

Theorem 2.5 (A Hermite-Biehler Theorem for Entire Functions, (cf. [Le, p. 315, Theorem $\left.\left.\left.4^{\prime}\right]\right)\right)$. Set

$$
\omega(z):=P(z)+i Q(z),
$$

where $P(z)$ and $Q(z)$ are nonconstant real entire functions. Then $\omega(z)$ is a function of class $\overline{H B}$ if and only if, for any real constants $\alpha$ and $\beta$, the entire function $\alpha P(z)+\beta Q(z)$ has only real zeros, and for some $x_{0} \in \mathbf{R}$

$$
Q^{\prime}\left(x_{0}\right) P\left(x_{0}\right)-Q\left(x_{0}\right) P^{\prime}\left(x_{0}\right)>0 \text {. }
$$


Remarks. If the entire function $\omega(z)$ (cf. 2.11) of class $\overline{\mathrm{HB}}$ has order less than 2, then it follows from Theorem 2.5 that $P(z)$ and $Q(z)$ are functions in the Laguerre-Pólya class. Moreover, it is known (cf. [Le, p. 314]) that inequality (2.12) can be replaced by the stronger condition

$$
Q^{\prime}(x) P(x)-Q(x) P^{\prime}(x) \geq 0 \quad \text { for all } x \in \mathbf{R} .
$$

\section{NeW RESUlts WITH APPLICATIONS}

We now introduce a geometric condition on the location of the zeros of the Fourier transform

$$
F(x ; K):=\int_{-\infty}^{\infty} K(t) e^{i x t} d t,
$$

where $K(t)$ is an admissible kernel, and we use Theorem 2.5 to generate $t w o$ families of entire functions in the Laguerre-Pólya class.

Proposition 3.1. Suppose that all the zeros of the real entire function $F(x ; K)$ (cf. (3.1)) lie in the strip

$$
S(\tau):=\{z \in \mathbf{C}:|\operatorname{Im} z|<\tau\} \quad \text { for some } \tau>0 .
$$

Then for each fixed $\mu \geq \tau$,

$$
P_{\mu}(x):=P_{\mu}(x ; K):=2 \int_{0}^{\infty} K(t) \cosh (\mu t) \cos (x t) d t \in \mathscr{L}-\mathscr{P}
$$

and

$$
Q_{\mu}(x):=Q_{\mu}(x ; K):=2 \int_{0}^{\infty} K(t) \sinh (\mu t) \sin (x t) d t \in \mathscr{L}-\mathscr{P} .
$$

Proof. Fix $\mu \geq \tau$. Then by Theorem 2.3, $F(z-i \mu ; K)$ is an entire function of order less than 2 , and it follows from the assumptions of the proposition that all the zeros of $F(z-i \mu ; K)$ lie in the open upper half-plane, $\operatorname{Im} z>0$.

From (3.1), we have

$$
F(z-i \mu ; K)=\int_{-\infty}^{\infty} K(t) e^{\mu t}(\cos (z t)+i \sin (z t)) d t,
$$

which, since $K(t)$ is an even function (cf. 2.4(iii)), can be expressed in the form

$$
F(z-i \mu ; K)=P_{\mu}(z)+i Q_{\mu}(z),
$$

where

$$
P_{\mu}(z):=\int_{-\infty}^{\infty} K(t) e^{\mu t} \cos (z t) d t=2 \int_{0}^{\infty} K(t) \cosh (\mu t) \cos (z t) d t,
$$

and

$$
Q_{\mu}(z):=\int_{-\infty}^{\infty} K(t) e^{\mu t} \sin (z t) d t=2 \int_{0}^{\infty} K(t) \sinh (\mu t) \sin (z t) d t
$$


Since $F(z ; K)$ is a real entire function of order strictly less than 2 , and since by hypcthesis all the zeros of $F(z ; K)$ lie in the strip $S(\tau)$, it is known (cf. [B, Theorem 6]) that there is a sequence $\left\{h_{n}(z)\right\}_{n=1}^{\infty}$ of real polynomials such that each $h_{n}(z)$ has all its zeros in $S(\tau)$, and such that

$$
\lim _{n \rightarrow \infty} h_{n}(z)=F(z ; K)
$$

uniformly on compact subsets of the complex plane $\mathbf{C}$. Thus, as $h_{n}$ is a polynomial, it is evident (cf. [Le, p. 307]) that for each $n(n=1,2,3, \cdots), h_{n}(z-i \mu)$ is a function of class $\overline{\mathrm{HB}}$ and that

$$
\lim _{n \rightarrow \infty} h_{n}(z-i \mu)=F(z-i \mu ; K),
$$

uniformly on compact subsets of $\mathbf{C}$. But the uniform limit (on compact subsets of C) of functions in class $\overline{\mathrm{HB}}$ is also a function in class $\overline{\mathrm{HB}}$ (cf. [Le, p. 314]), and therefore $F(z-i \mu ; K) \in \overline{\mathrm{HB}}$. Hence, by the remarks following Theorem 2.5, $P_{\mu}(z)$ and $Q_{\mu}(z)(\mu \geq \tau)$ are functions in the Laguerre-Pólya class.

As a direct consequence of the foregoing proof, the integral representations of (3.3) and (3.3'), and inequality (2.13), we obtain the following corollary.

Corollary 3.2. If all the zeros of the real entire function $F(x ; K)$ (cf. (3.1)) lie in the strip $S(\tau)$ (cf. (3.2)), then for all $x \in \mathbf{R}$ and $\mu \geq \tau$

$$
\int_{0}^{\infty} \int_{0}^{\infty} K(t) K(s) \sinh (\mu s) \cosh (\mu t) A(x, s, t) d t d s \geq 0,
$$

where

$$
A(x, s, t):=s \cos (x s) \cos (x t)+t \sin (x s) \sin (x t) .
$$

Remarks. We remark that Corollary 3.2 is only one of several consequences of Proposition 3.1 and Theorem 2.5. Indeed, under the hypotheses of Proposition 3.1, we know from Theorem 2.5 that for any real constants $\alpha$ and $\beta$, the function $\alpha P_{\mu}(x)+\beta Q_{\mu}(x)$, where $\mu \geq \tau$, belongs to the Laguerre-Pólya class. Hence, in particular, $\alpha P_{\mu}(x)+\beta Q_{\mu}(x)$ satisfies the Laguerre inequalities of (2.2).

Our next result, while elementary in character, is the crucial fact needed, in conjunction with the foregoing results, to prove Theorem 3.4. The proof of Lemma 3.3 will be omitted, however, since it merely involves the verification of the given formulas. (Note that differentiation under the integral sign with respect to $x$ in (2.5) is readily justified since $K(t)$ is an admissible kernel.)

Lemma 3.3. If $K(t)$ is an admissible kernel, then for all $x, y \in \mathbf{R}$

$$
\Delta(x, y ; K)=8 \int_{0}^{\infty} \int_{0}^{\infty} K(t) K(s) \Psi(x, y, t, s) d t d s,
$$

where $\Delta(x, y ; K)$ is defined by (2.8) and

$$
\begin{aligned}
& \Psi(x, y, t, s):= \\
& \quad s^{2}[\cos (x t) \cos (x s) \cosh (y t) \cosh (y s)+\sin (x t) \sin (x s) \sinh (y t) \sinh (y s)] \\
& \quad+s t[\cos (x t) \cos (x s) \sinh (y t) \sinh (y s)+\sin (x t) \sin (x s) \cosh (y t) \cosh (y s)] .
\end{aligned}
$$


Moreover, for each $x, y \in \mathbf{R}$, the double integral in (3.7) can be expressed as

$$
\frac{1}{8} \Delta(x, y ; K)=L\left(P_{\mu}(x)\right)+L\left(Q_{\mu}(x)\right) \quad(y=\mu),
$$

where

$$
\begin{gathered}
L\left(P_{\mu}(x)\right):=\left[P_{\mu}^{\prime}(x)\right]^{2}-P_{\mu}(x) P_{\mu}^{\prime \prime}(x) \quad(x \in \mathbf{R}), \\
L\left(Q_{\mu}(x)\right):=\left[Q_{\mu}^{\prime}(x)\right]^{2}-Q_{\mu}(x) Q_{\mu}^{\prime \prime}(x) \quad(x \in \mathbf{R}),
\end{gathered}
$$

and $P_{\mu}(x)$ and $Q_{\mu}(x)$ are defined by (3.3) and $\left(3.3^{\prime}\right)$ respectively.

With the aid of Lemma 3.3, we next prove the following theorem.

Theorem 3.4. Suppose that all the zeros of $F(x ; K)(c f$. (3.1)) lie in the strip $S(\tau)$ (cf. (3.2)) for some fixed $\tau>0$. Then $F(x ; K) \in \mathscr{L}-\mathscr{P}$ if and only if

$$
\Delta(x, y ; K) \geq 0 \quad \text { for } 0<x<\infty \text { and } 0 \leq y<\tau,
$$

where $\Delta(x, y ; K)$ is defined by $(2.8)$.

Proof. The necessity of (3.12) is clear from (2.7) of Theorem 2.4. To prove the sufficiency, suppose that $\mu \geq \tau$, and consider the functions $P_{\mu}(x)$ and $Q_{\mu}(x)$ defined by (3.3) and $\left(3.3^{\prime}\right)$, respectively. Then by (3.9)

$$
\frac{1}{8} \Delta(x, \mu ; K)=L\left(P_{\mu}(x)\right)+L\left(Q_{\mu}(x)\right),
$$

where $L\left(P_{\mu}(x)\right)$ and $L\left(Q_{\mu}(x)\right)$ are given by (3.10) and (3.11). Now, by Proposition $3.1, P_{\mu}(x), Q_{\mu}(x) \in \mathscr{L}-\mathscr{P}$ for all $\mu \geq \tau$, and consequently $P_{\mu}(x)$ and $Q_{\mu}(x)$ satisfy the following Laguerre inequalities with $m=1$ (cf. (2.2), (3.10) and (3.11)):

$$
L\left(P_{\mu}(x)\right) \geq 0 \quad \text { and } \quad L\left(Q_{\mu}(x) \geq 0 \quad(x \in \mathbf{R}, \mu \geq \tau) .\right.
$$

From (3.7) and (3.8) we see that

$$
\left\{\begin{aligned}
\Delta(x, y ; K) & =\Delta(-x, y ; K) \quad(x, y \in \mathbf{R}) \\
& \text { and } \\
\Delta(x, y ; K) & =\Delta(x,-y ; K) \quad(x, y \in \mathbf{R})
\end{aligned}\right.
$$

and thus by (3.14) and (3.15)

$$
\Delta(x, y ; K) \geq 0 \quad \text { for all }|y| \geq \tau \text { and } x \in \mathbf{R} .
$$

Finally, it follows from (3.12), (3.15) and (3.16) that $\Delta(x, y ; K) \geq 0$ for all $x, y \in \mathbf{R}$, and hence, by Theorem $2.4, F(x ; K) \in \mathscr{L}-\mathscr{P}$.

We conclude this paper with an application to the Riemann $\xi$-function $\xi(x)$, where

$$
\xi\left(\frac{x}{2}\right):=8 \int_{0}^{\infty} \Phi(t) \cos (x t) d t
$$


and where

$$
\Phi(t):=\sum_{n=1}^{\infty}\left(2 n^{4} \pi^{2} e^{9 t}-3 n^{2} \pi e^{5 t}\right) \exp \left(-n^{2} \pi e^{4 t}\right) .
$$

For simplicity of notation, we set

$$
F(x ; \Phi):=\int_{-\infty}^{\infty} \Phi(t) e^{i x t} d t
$$

Now, it is well-known (see, for example, [CNV, Theorem A]) that $\Phi(t)$ satisfies the properties (2.4), and hence $\Phi(t)$ is an admissible kernel. Moreover, it is easy to check that $\xi(x / 2) \in \mathscr{L}-\mathscr{P}$ if and only if $F(x ; \Phi) \in \mathscr{L}-\mathscr{P}$. Now the Riemann $\xi$-function is related to the Riemann zeta-function by the formula (cf. [P2, p. 10])

$$
\xi(i z)=\frac{1}{2}\left(z^{2}-\frac{1}{4}\right) \pi^{-z / 2-1 / 4} \Gamma\left(\frac{z}{2}+\frac{1}{4}\right) \zeta\left(z+\frac{1}{2}\right) .
$$

Thus, as the nontrivial zeros of $\zeta(z)$ lie in the critical strip $0<\operatorname{Re} z<1$ (cf. [T, p. 30]), it follows that the zeros of $\xi(x / 2)$ lie in $S(1)$ (cf. (3.2)), and a direct application of Theorem 3.4 yields the following corollary.

Corollary 3.5. If $K(t):=\Phi(t)$, then $F(x ; \Phi) \in \mathscr{L}-\mathscr{P}$ if and only if

$$
\Delta(x, y ; \Phi) \geq 0 \quad \text { for } 0<x<\infty \text { and } 0 \leq y<1,
$$

where $\Delta(x, y ; K)$ is defined by $(2.8)$.

To underscore the significance of Corollary 3.5, we compare (2.7) and (3.21) and observe that the Riemann Hypothesis is valid if and only if inequality (3.21) holds.

\section{REFERENCES}

[AK] N. I. Ahiezer and M. G. Krein, Some questions in the theory of moments, Transl. Math. Mono., vol. 2, Amer. Math. Soc., Providence, R.I., 1962.

[B] N. G. de Bruijn, The roots of trigonometric integrals, Duke Math. J. 17 (1950), 197-226.

[CNV] G. Csordas, T. S. Norfold and R. S. Varga, The Riemann Hypothesis and the Turán inequalities, Trans. Amer. Math. Soc. 296 (1986), 521-541.

[CV1] G. Csordas and R. S. Varga, Moment inequalities and the Riemann Hypothesis, Constr. Approx. 4 (1988), 175-198.

[CV2] __, Integral transforms and the Laguerre-Pólya class, Complex Analysis, (to appear).

[CV3] _. Necessary and sufficient conditions and the Riemann Hypothesis, (to appear).

[J] J. L. W. V. Jensen, Recherches sur la théorie des équations, Acta Math. 36 (1913), 181-195.

[L] E. Laguerre, Oeuires, vol. I, Gauthier-Villars, Paris, 1898.

[Le] B. Ja. Levin, Distribution of zeros of entire functions, Transl. Math. Mono., vol. 5, Amer. Math. Soc., Providence, R.I., 1964; rev. ed., 1980.

[M] N. N. Meiman, On the distribution of zeros of an integral function (Russian), Dokl. Akad. Nauk SSR 40 (1943), 179-181.

[P1] G. Pólya, Üher trigonometrische Integrale mit nur reellen Nullstellen, J. Reine Angew. Math. 158 (1927), 6-18. 
[P2] __ Über die algebraisch-funktionentheoritischen Untersuchungen von J. L. W. V. Jensen, Kgl. Danske Vid. Sel. Math.-Fys. Medd. 7 (1927), 3-33.

[P3] _ Collected papers, vol. II: Location of zeros, R. P. Boas, ed., MIT Press, Cambridge, 1974.

[S] H. Skovgaard, On inequalities of the Turán type, Math. Scand. 2 (1954), 65-73.

[T] E. C. Titchmarsh, The theory of the Riemann zeta function, second edition (revised by D. R. Heath-Brown), Oxford Science Publications, 1986.

Department of Mathematics, University of Hawail at Manoa, Honolulu, Hawail 96822 Institute for Computational Mathematics, Kent State University, Kent, Ohio 44242 